\title{
SIMPACTECTOMIA LOMBAR RETROPERITONEOSCÓPICA PARA TRATAMENTO DA HIPER-HIDROSE PLANTAR
}

\section{RETROPERITONEOSCOPIC LUMBAR SYMPATHECTOMY FOR THE TREATMENT OF PLANTAR HYPERIDROSIS}

\author{
Marcelo de Paula Loureiro, TCBC-PR'; Neomar Roman²; Sheila Cristina Weigmann³;
} Aline Fontana ${ }^{3}$; Paulo César Bufara Boscardim ${ }^{4}$

\begin{abstract}
RESUMO: Objetivo: Observar o seguimento de até quatro anos de mulheres submetidas à simpatectomia lombar por retroperitonioscopia, procurando identificar seus efeitos colaterais, bem como sua efetividade no controle da hiper-hidrose plantar. Métodos: Entre junho/2002 e julho2006, operamos 44 pacientes com hiperidrose plantar. Todas apresentavam hiper-hidrose plantar persistente pós simpatectomia torácica com exceção de uma única paciente com diagnóstico de hiperidrose plantar primária. Resultados: O seguimento médio de 22,3 meses, mostra grande satisfação referida pela maioria das pacientes e piora do suor compensatório em $37 \%$ delas. Não houve relato de alteração sexual nesta casuística. Conclusão: A simpatectomia lombar por retroperitonioscopia é uma técnica segura, eficaz e associada a e aceitáveis efeitos colaterais, quando empregada no tratamento da hiperidrose plantar em mulheres jovens (Rev. Col. Bras. Cir. 2007; 34(4): 222-224).
\end{abstract}

Descritores: Simpatectomia/ métodos; Hiperidrose/ cirurgia; Laparoscopia.

\section{INTRODUÇÃO}

O efeito do sistema nervoso simpático sobre o tônus vascular foi inicialmente observado por Du Petit em $1727^{1} \mathrm{e}$ serviu de base lógica para o desenvolvimento de procedimentos que visassem a simpatectomia.

A simpatectomia lombar foi inicialmente descrita em Buenos Aires no ano de 1924, por Julio Diez ${ }^{2}$ e no mesmo ano por Royle na Austrália ${ }^{3}$. No início era utilizada para tratamento das alterações isquêmicas e dolorosas dos membros inferiores, e assim permaneceu por várias décadas, gozando de grande popularidade. A partir dos anos 60, com a introdução das técnicas de reconstrução arterial, seu uso foi diminuindo lentamente. Hoje em dia, ainda é útil em pacientes selecionados, com doença arterial, porém sua principal indicação é para o tratamento da hiper-hidrose plantar.

Como a maior parte dos doentes com hiperhidrose plantar permanecem com o problema após a simpatectomia torácica, aumenta a cada dia a demanda pela simpatectomia lombar retroperitoneoscópica. Os efeitos de uma ampla supressão do sistema simpático, com duas simpatectomias bilaterais num mesmo indivíduo ainda são especulativos. Apresentamos uma série de 43 pacientes submetidos a simpatectomia lombar retroperitoneoscópica (SLR) após terem sido submetidos à simpatectomia torácica , e sua evolução com seguimento de até quatro anos.

\section{MÉTODOS}

Entre Junho de 2002 e Julho de 2006 , 44 pacientes foram submetidas a simpatectomia lombar retroperitoneoscópica, todas do sexo feminino, com idade entre 16 e 39 anos(média de 23,7 anos). Não foram operados pacientes do sexo masculino devido ao possível efeito colateral da ejaculação retrógrada. Todas apresentavam hiperidrose plantar persistente pós simpatectomia torácica com exceção de uma única paciente com diagnóstico de hiperidrose plantar primária.

O intervalo entre a simpatectomia torácica e a lombar variou de dois meses a cinco anos. Em 38 das pacientes, optou-se por aguardar ao menos seis meses para realizar a operação, uma vez que a melhora da hiperidrose plantar após a simpatectomia torácica pode ocorrer neste intervalo de tempo.

Foram excluídas apenas duas das pacientes por perda de seguimento. As demais foram acompanhadas por um período que variou entre 1 e 49 meses (média de 22,3 meses). Os dados do último seguimento foram obtidos por meio de entrevista telefônica. Nesta pesquisou-se o grau de satisfação com o tratamento cirúrgico, o aparecimento ou eventual piora da hiper-hidrose compensatória quantificada por meio de escala analógica de 1 a 10 (1 mínima e 10 péssima) e os possíveis efeitos colaterais sexuais como alteração da libido.

\footnotetext{
1. Coordenador do Curso de Pós-Graduação em Cirurgia Minimamente Invasiva do UnicenP.

2. Cirurgião Vascular.

3. Doutoranda de Medicina da UFPR. Cirurgião Torácico do HC da UFPR.

4. Cirurgião toráxico do HC da UFPR.

Recebido em 05/12/2006

Aceito para publicação em 07/02/2007

Conflito de interesses: nenhum

Fonte de financiamento: nenhuma
}

Trabalho realizado no Curso de Pós Graduação da Universidade Positivo ( UnicenP) e Hospital Santa Cruz de Curitiba. 


\section{RESULTADOS}

Não houve conversão para a técnica aberta em nenhum dos casos. Das 88 simpatectomias lombares, apenas uma não foi efetiva. A paciente manteve seu tônus simpático, com hiper-hidrose à direita e passou a apresentar parestesia no território de inervação do nervo genito-femoral, que foi seccionado inadvertidamente.

O tempo da operação variou de 2,5 horas, nos primeiros casos, a 50 minutos. Não houve lesão vascular e em apenas duas pacientes os vasos lombares finalizados?

A lesão do peritôneo ocorreu em 16 das 44 pacientes, mas não impediu a realização do procedimento. Uma das pacientes apresentava situs inversus totalis, o que também não acrescentou maior dificuldade para a realização da cirurgia.

A drenagem pós-operatória foi necessária apenas na primeira paciente desta série. Não foram observados hematomas ou seromas em nenhuma das pacientes operadas.

O tempo de internação hospitalar foi de um dia para 40 pacientes e de dois dias para quatro pacientes. Para analgesia pós-operatória foram utilizados anti-inflamatórios pelo período de sete dias.

Houve uma reoperação, realizada por retroperitoneoscopia, na paciente cujo nervo simpático havia sido confundido com o nervo genito-femoral.

O seguimento tem sido realizado semestralmente. Apenas duas das pacientes , já no primeiro ano de pós-operatório, tiveram seu seguimento descontinuado e foram excluídas do estudo.

No último contacto, por telefone, 42 das 44 responderam algumas perguntas dirigidas.

A hiper-hidrose compensatória após a simpatectomia torácica e antes da simpatectomia lombar estava presente em $37(88 \%)$ das 42 pacientes entrevistadas, atingindo principalmente o abdômen e as costas . Ela era considerada leve em 25, moderada em 10 e intensa em duas .

A hiper-hidrose compensatória não piorou após a simpatectomia lombar em 27 pacientes, sendo que, três consideraram que houve inclusive melhora, nas pernas e na região inguinal especialmente. Quinze referiram piora da hiperidrose compensatória após a SL. Esta piora foi classificada em escala ordinal, em Graus de 1 a 10 considerando-se 10 como sendo piora de $100 \%$. Desta forma quatro pacientes consideraram piora Grau 1; três pacientes Grau 2; quatro pacientes Grau 3; duas pacientes Grau 5; e duas pacientes Grau 8. Assim 63\% das pacientes ou não apresentaram piora da HH compensatória ou até melhoraram.

A melhora da hiper-hidrose plantar ocorreu em todas as pacientes. Quando questionadas para graduá-la de 1 a 10, duas consideraram-na Grau 7; duas Grau 8; duas Grau 9 e 36 Grau 10.

Duas pacientes referiram aumento do suor palmar, transitório em uma delas. A outra, apesar do suor persistente, não o considerou sigificativo.

O nível de satisfação com a cirurgia também foi questionado e considerado uniformemente como $100 \%$.

Algumas pacientes relacionaram à operação o surgimento de varicoses (três), parestesia na perna direita (duas), maior suscetibilidade ao frio (duas), distensão abdominal (três), intolerância a alimentos doces (uma) e leve hipotensão postural (duas). Nenhuma das pacientes referiu efeitos colaterais, sexuais após a cirurgia.

Finalmente, todas recomendariam a operação para quem dela necessitasse.

\section{DISCUSSÃo}

Com o desenvolvimento da cirurgia minimamente invasiva, novas técnicas de acesso ao retroperitônio têm se mostrado eficazes para a abordagem das estruturas aí locali$\operatorname{zadas}^{4,5,6}$. A primeira série de simpatectomia lombar laparoscópica extraperitoneai foi publicada por Hourlay em $1995^{7}$. Desde então a técnica têm se mostrado segura nas mãos de cirurgiões laparoscópicos experientes ${ }^{8}$.

A hiper-hidrose é a indicação mais freqüente para a realização das simpatectomias, tanto torácica quanto lombar ${ }^{9}$. É uma doença que atinge cerca de $1 \%$ da população e se caracteriza pelo suor em excesso em determinadas regiões como as mãos, a face, as axilas e os pés. A simpatectomia torácica endoscópica cura a hiper-hidrose acima da linha da cintura, porém seus resultados para o suor em excesso nos pés não são tão expressivos promovendo melhora em até $58 \%$ dos $\operatorname{casos}^{8}$. Muitos dos pacientes que são submetidos à simpatectomia torácica para controle da hiper-hidrose permanecem com ou pioram da doença nos pés. Além disto, uma complicação freqüente da simpatectomia torácica é o desenvolvimento do suor reflexo ou compensatório, que atinge de 20 a $80 \%$ dos operados ${ }^{9-12}$.

A simpatectomia lombar é eficaz no tratamento da hiper-hidrose plantar isolada ou persistente após a simpatectomia torácica ${ }^{13,14}$. Entretanto existe uma grande preocupação com o efeito da simpatectomia lombar no suor reflexo ou compensatório já desencadeado pela simpatectomia torácica. A expectativa de piora desta condição, que muitas vezes já é debilitante ao paciente, poderia configurar uma contra-indicação à própria simpatectomia lombar.

Pode-se observar que apesar da piora da hiperhidrose compensatória em alguns dos pacientes, ela não foi significativa na maioria delas. Estes dados carecem de discussão na literatura. Mesmo na eventualidade de grande piora da compensação do suor, não parece haver uma piora proporcional na qualidade de vida das pacientes, que permanecem muito satisfeitas com o resultado da simpatectomia lombar. A eliminação do suor plantar tende a suplantar o inconveniente da piora da hiper-hidrose compensatória.

Goldstein, revisando as complicações de 791 pacientes submetidos a simpatectomia lombar aberta refere $10 \%$ de complicações específicas, como abolição da ejaculação em 22 pacientes e causalgia em 41, morbidade geral de $0,6 \%$ (TEP e hemorragias) e mortalidade de $0,6 \%$ (arritmias, descompensação cardíaca e hemorragias) ${ }^{15}$.

Em nossa casuística as complicações sexuais tais como: alteração de libido, lubrificação vaginal, dispareunia e frigidez não foram referidas.

A simpatectomia lombar retroperitoneoscópica é uma técnica segura e eficaz no tratamento da hiperidrose plantar, e está associada a poucos e aceitáveis efeitos colaterais. 


\begin{abstract}
Background: Describe a 4-years follow-up offemales submitted to lumbar sympathectomy for hiperhidrosis. Methods: From June 2002 to July 2006, we have operated 44 patients with plantar hyperidrosis. Only one of them had primary plantar hyperidrosis. The others had persistent plantar hyperidrosis after thoracic sympathectomy. Results : We show our results with a median follow up of 22.3 months. Most patients were very satisfied and only $37 \%$ had worsened their compensatory sweat. Conclusion: Laparoscopic lumbar sympathectomy is a safe and effective technique which is associated with few and acceptable collateral effects, when it is indicated in the treatment of plantar hyperhidrosis in young women.
\end{abstract}

Key words: Sympathectomy/methods; Hyperhidrosis /surgery; Laparoscopy.

\section{REFERÊNCIAS}

1. Petit FP. Mémoire dans lequel il est démontré que les nerfs intercosteaux fournissent des rameaux qui portent des esprits dans les yeux. Histoire de l'Académie royale des sciences; Avec les Mémoires de Mathématique \& de Physiologie pour la même année. 1727: 1-19.

2. Diez J. Um nuevo método de simpatectomía periférica para el tratamiento de las afecciones tróficas y gangrenosas de los miembros: la disociación fascicular. Bol Soc Cir Buenos Aires. 1924; 8:792-4.

3. Royle ND. A new operative procedure in the treatment of spastic paralysis and its experimental basis. Med J Aust. 1924; 1(4):7786.

4. Kathouda N, Wattanasirichaigoon S, Tang E, Yassini P, Ngaorungsri U. Laparoscopic lumbar sympathectomy. Surg Endosc. 1997; 11(3):257-60.

5. Gill IS, Clayman RV, Albala DM, Aso Y, Chiu AW, Das S, Donovan JF, Fuchs GJ, Gaur DD, Go H, Gomella LG, Grune MT, Harewood LM, Janetschek G, Knapp PM, McDougall EM, Nakada SY, Preminger GM, Puppo P, Rassweiler JJ, Royce PL, Thomas R, Urban DA, Winfield HN. Retroperitoneal and pelvic extraperitoneal laparoscopy: an international perspective. Urology. 1998; 52(4):566-71.

6. Watarida S, Shiraishi S, Fujimura M, Hirano M, Nishi T, Imura M, Yamamoto I. Laparoscopic lumbar sympathectomy for lower limb disease. Surg Endosc. 2002; 16(3):500-3. Epub 2001 Nov 16.

7. Hourlay P, Vangertruyden G, Verduyckt F, Trimpeneers F, Hendrickx J. Endoscopic extraperitoneal lumbar sympathectomy. Surg Endosc. 1995; 9(5):530-3.

8. de Campos JR, Kauffman P, Werebe Ede C, Andrade Filho LO, Kusniek S, Wolosker N, Jatene FB. Quality of life, before and after thoracic sympathectomy: report on 378 operated patients. Ann Thorac Surg. 2003; 76(3):886-91
9. Hsia JI, Chen CY, Hsu CP, Shai SI, Yang SS. Outpatient thoracoscopic limited sympathectomy for hyperhidrosis palmaris. Ann Thorac Surg. 1999; 67(1):258-9.

10. Gossot D, Toledo L, Fritsch S, Célérier M. Thoracoscopic sympathectomy for upper limb hyperhidrosis: looking for the right operation. Ann Thorac Surg. 1997; 64(4):975-8.

11. Plas EG, Függer R, Herbst F, Fritsch A. Complications of endoscopic thoracic sympathectomy. Surgery. 1995; 118(3):493-5.

12. Yamamoto H, Kanehira A, Kawamura M, Okada M, Ohkita Y. Needlescopic surgery for palmar hyperhidrosis. J Thorac Cardiovas Surg. 2000; 120(2):276-9.

13. Tseng MY, Tseng JH. Endoscopic extraperitoneal lumbar sympathectomy for plantar hyperhidrosis: case report. J Clin Neurosci. 2001; 8(6):555-6.

14. Moran KT, Brady MP. Surgical management of primary hyperhidrosis. Br J Surg. 1991; 78(3):279-83.

15. Goldstein M, Ectors P, Dereume JP, van der Stricht J. [Complications of lumbar sympathectomy. Retrospertive study of 791 patientes]. Acta Chir Belg. 1977; 76(1):739 .

Como citar este artigo:

Loureiro MP, Roman N, Fontana SCW, Boscardim PCB. Simpactectomia lombar retroperitonioscópica para tratamento da hiper-hidrose plantar. Rev Col Bras Cir [periódico na Internet]. 2007;34(3). Disponível em URL:http://www.scielo.br/rcbc

Endereço para correspondência:

Dr. Marcelo de Paula Loureiro

Rua Vereador Constante Pinto 195, ap. 62

Curitiba, Paraná, Brasil

CEP: 82510-240

E-mail: mloureiro@unicenp.edu.br 